\title{
African American Childhood and Adolescent Obesity: Interventions with Group Therapy
}

\section{Arthur Horton *}

Department of Psychology, Lewis University, USA

*Corresponding author: Arthur Horton, Department of Psychology, Lewis University, USA, Tel: +8158365314; E-mail: ahorton756@aol.com

Received date: September 27, 2017, Accepted date: September 29, 2017, Published date: October 04, 2017

Copyright: @ 2017 Horton A. This is an open-access article distributed under the terms of the Creative Commons Attribution License, which permits unrestricted use, distribution, and reproduction in any medium, provided the original author and source are credited.

\section{Short Communication}

Obesity rates tend to be higher in Black children. Nationally, in 2012, 20.5\% of African-American girls were obese compared to $15.6 \%$ of White girls and $19 \%$ of African-American boys were obese compared to $12.6 \%$ of white Boys. For youth from 2 years old to 19 years old, $8 \%$ of African-American children are considered severely obese, with the severe weight gain occurring most often between the third and eighth grades. African Americans are seriously eating themselves into the grave. This is a conversation often held with the late Dick Gregory, but I never agreed with his extremities on eating either. The key is to be healthy and balanced. The key is nutrition and it should be taught in schools, as to what foods are healthy and what foods are not. Many churches haves taken it upon themselves to teach good, healthy habits.

"Remember. We all stumble, every one of us. That's why it's a comfort to go hand in hand" Emily Kimbrough quotes. Individuals have disagreements that cause hard times within their life. Gratefully, there are solutions to help individuals overcome the problems that are obesity related and the relationships with others. There are multiple types of group therapies that may help. Psycho education and confrontation will be examined regarding the strengths of each therapy in regards to help better the relationships between individuals and weight related problems or addictions using group therapy

One approach to handling conflict for an individual would be Psycho education. Psycho education group therapy is based on specific disorders such as anxiety, depression, and eating disorder. The purpose of Psycho education group therapy is to teach the individual going through certain psychological crisis about their particular condition such as depression, and help to reduce symptoms from occurring again. Being in a group allows the individual to meet and discuss their issues with others who go through the same difficulties with their disorder. Some people believe a disadvantage with this Psycho education group therapy to be a resolution is forcing an individual to talk about their struggles with a disorder, but in fact the individual is in charge of controlling the information being shared with others. While using Psycho education therapy the individual usually meets with the group weekly. Each meeting is facilitated by a group leader. The group leader can be a psychologist or psychiatrist. The group leader works to make the setting safe and comforting for the group. Each session will focus on a certain topic. When a topic is chosen for the session, the session will discuss how the individual is affected as well as the environmental setting that the individual is usually in. A way to engage the individuals in the discussion and to have a group benefit from understanding is the use of role playing as a technique. Within Psycho education therapy, individuals learn to respect each other and learn to keep the information told by others within the meetings. Some of the overall benefits of this type of group therapy are for an individual to learn about their conditions as well as skills to help manage their disorder. Individuals may learn exercises and techniques in order to deal with their disorder in stressful situations or environments. This type of therapy also teaches the individual, they are not alone. The individual learns that others are going through issues as they are. The individuals come to learn they have a safe place to be with others who understand their struggles [1].

An example topic used in Psycho education therapy may be Bipolar. Bipolar is a complex illness. There are multiple symptoms and types. The most common symptoms of Bipolar are dramatic and unpredictable mood swing. There are cases of Bipolar that range from mild to severe [2]. Within a specific study, Bipolar was the main focuses while using Psycho education group therapy to help individuals cope with their disorder. Within the study the individuals worked on specific goals. "Bipolar disorder is estimated to affect between $0.3 \%$ and $1.5 \%$ of individuals worldwide". With the use of Psycho education group therapy a nurse, a social worker, and psychiatrist worked to help the individuals identify signs and symptoms of depression and mania. Identifying gave knowledge about the course of illness and what risk factors could possibly lead to relapse. The group session also helped raise awareness concerning the impact bipolar on psychological, cognitive, physical, emotional, and social functioning. The leaders also helped individuals understand major pharmacologic treatment modalities for Bipolar and common side effects. The group sessions provided information on guidelines regarding medication effectiveness and safety as well as information on cognitive strategies pertaining to Bipolar. The overall goal of the study was to increase self-confidence, self-awareness, and social skills through interaction with other group members [3].

In addition to Psycho education being a solution to individuals dealing with disorders within groups, confrontation group therapy may also be an option. Therapeutic confrontation is a process by which a therapist provides direct, reality, oriented feedback to an individual regarding the individuals own thoughts, feelings or behavior. Confrontation may vary in timing, intensity, emotional content, accompanying interventions, and the relationship and organizational contexts it occurs. Therapeutic confrontation was founded on four assumptions. The first assumption was addiction is rooted in an immature, defective character encased within an armor-plated defense structure. The passive methods of traditional psychotherapies are ineffective in penetrating defensive structures and altering deformity of character of an individual. Also, the individual can be reached only by a dynamite charge that breaks through their protective shield. Lastly, verbal confrontation is the most effective means of engaging and changing addictive behavior. Confrontation group therapy usually deals with individuals facing addiction of alcohol, narcotics and compulsive overeating [4]. 
Citation: Horton A (2017) African American Childhood and Adolescent Obesity: Interventions with Group Therapy. J Gen Pract (Los Angel) 5:

Page 2 of 2

An example of the use of confrontational therapy would be in the case of the actor Charlie Sheen having an addiction to drugs and alcohol. CNN provides an article that Charlie battles with the cocaine and alcohol use. He has relapsed multiple times. In order for confrontation therapy to work, the addicts needs to first realize what they were doing before doesn't work. William Moyers (vice president of foundation relations at Hazelden Foundation) quotes, "What he should be doing is taking very seriously his problem, not trying to compartmentalize it or minimize it or trivialize it, but really to recognize that his is probably a problem of the mind, the body and the spirit". The article discusses group therapy puts a patient into an environment of people who are both empathetic and who are also working toward recovery. Also, Hearing the experiences of other people gives hope and helps an individual with their healing process. Moyers again states, "At the end of the day, (Sheen's) ability to recover depends on his willingness to be part of a larger experience, and that larger experience will include group therapy and recovery meetings." The group environment provides an opportunity for caring confrontation from peers and the knowledge that others can understand the problems an individual faces rather than facing the shame of isolation [5].

Overall, confrontation involves a therapist directing an individual's attention to their behavior and thought process. Confrontation is used to help a patient analyze resistance, to aid in the identification of defences, and help the individual understand that they are resisting, why they are resisting, and how they are resisting dealing with their addiction in order to change their habits in hope to lead away from continuing relapsing [6].

Every individual is different therefore therapy can work for some and not for others. Each person deals with issues differently as well as adapting to change regarding disorders and addictions. Psycho education may help some individuals while Confrontation al therapy works for others due to the specific problems each individual has regarding disorders or addiction [7].

\section{References}

1. Gromisch E (2012) Explore the benefits of psychoeducation group therapy. Bright Hub Education.

2. Bipolar Disorder health center (2005) WebMD.

3. Michalak EE, Yatham LN, Wan DD, Lam RW (2005) Perceived quality of life in patients with bipolar disorder. Does group psychoeducation have an impact? Can J Psychiatry 50: 95-100.

4. DiGiacomo TM (2010) The effective use of confrontation in family therapy: A process study. Clin psychol 1-66.

5. Landau E (2011) Addiction relapse: Part of chronic illness.

6. Confrontation in addiction treatment (2007) Cafety: Community alliance for the ethical treatment of you.

7. Quotations about relationships (1998-2013) Welcome to the quote garden. 